\section{Shared care in} glaucoma: a national study of secondary care lead schemes in England

SA Vernon and A Adair

\begin{abstract}
Purpose To determine the number and nature of shared care schemes for glaucoma and glaucoma suspects operating in England. Methods A two-stage investigational process targeting all secondary-care ophthalmic departments with junior medical staff. An initial telephone contact for basic data (March 2006) was followed by a detailed questionnaire for completion by the scheme lead (May 2006).

Results The telephone contact showed that of the 131 eligible ophthalmic departments, 76 claimed to be operating a glaucoma-based shared care scheme. Questionnaires were returned from 74 of the 76 departments claiming to run a scheme, showing that there were only 66 schemes operating in mid 2006 in association with 62 departments. Of these, 14 were community-based (predominantly run by trained optometrists) and 52 operated 'in-house' (predominantly involving nurses and optometrists). Most schemes were $<6$ years old and of the 30 schemes seeing new patients, 14 (47\%) did not use gonioscopy as part of the assessment. In 8 schemes (12\%), the shared care staff members were able to prescribe medication for glaucoma.

Eye (2010) 24, 265-269; doi:10.1038/eye.2009.118; published online 5 June 2009

Keywords: glaucoma; shared care; review; England

Introduction

The Department of Health has recently published its review of the eye care services pathway pilot schemes, of which four involved glaucoma ${ }^{1}$. The new care pathways ${ }^{2}$ rely heavily on what is termed as 'shared care,' which can be defined as the devolvement of clinical management responsibilities to non-medical staff ${ }^{3}$. This may take place within the hospital environment ('in-house') or outside the hospital ('communitybased'). In the absence of data regarding the status of shared care within glaucoma management in the United Kingdom, we designed and carried out an investigation in the form of a telephone survey with a secondary postal questionnaire aiming to determine the number and management practices of the currently operating glaucoma shared care schemes in England. To our knowledge, this is the first wide-ranging review of any aspect of shared care within ophthalmology in the United Kingdom.
\end{abstract}

Conclusion In England, even before the outcome of the Department of Health shared care pilots had been published, $\sim 50 \%$ of ophthalmic departments were running shared care schemes for glaucoma. However, most schemes contributed only modestly to the overall volume of glaucoma care, indicating that the majority of glaucoma-related consultations still occur directly with ophthalmologists. The Royal College of Ophthalmologists guidelines on gonioscopy are not being followed in almost half of the schemes seeing new patients.

\section{Materials and methods}

Using the current RCOphth (Royal College of Ophthalmologists) directory of training schemes $(2006)^{4}$, we identified all departments within England that train junior ophthalmologists. Each department was contacted by telephone in March-April 2006 by an experienced research assistant (AA) who attempted to identify and then contact one of the following personnel (in order of preference): (1) glaucoma specialist consultant, (2)
Department of Ophthalmology, University Hospital, Nottingham, UK

Correspondence: SA Vernon,

Department of Ophthalmology, Queen's medical Centre, University Hospital, Derby Rd,

Nottingham, Notts NG7 2UH, UK

Tel: + 441159249924

Ext 63200;

Fax: +44 1159709749

E-mail: Stephen.Vernon@ nuh.nhs.uk

Received: 2 August 2008 Accepted in revised form: 4 April 2009

Published online: 5 June 2009

Results presented at UKEGS 2006 and RCOphth Congress 2007 
consultant with a special interest in glaucoma, or (3) business manager

AA then asked the contact whether a shared care scheme(s) in glaucoma was operated by the department and, if so, which staff shared the care and whether the scheme was 'in-house' (operated within the hospital environment) or 'in the community' (operated outside the confines of the hospital). The contact was then asked whether they would complete a detailed questionnaire.

In May 2006, the questionnaire was sent to all contacts (see Supplementary Information). In brief, details of the operational aspects of the scheme, namely the number and nature of the employed staff, length of training, the duties and decisions they were expected to make, number of patients seen per session by the staff, the percentage of glaucoma care delivered through the shared care route in the catchment area and the nature of medical input, including audit, were requested. The schemes were categorised by the professional status of the operational staff and data recorded on a computer database for subsequent analysis.

\section{Results}

\section{Initial telephone survey}

A total of 131 departments were identified from the RCOphth training handbook, all of which were able to provide initial data on whether they ran a shared care scheme in glaucoma. Of these, $76(58 \%)$ claimed to be operating a shared care scheme. A further seven (5\%) stated that they were 'working towards one'. Sixty-one (80\%) of the departments with a scheme stated they had an in-houseonly scheme, $9(12 \%)$ had a community scheme only and 6 $(8 \%)$ had both community and in-house schemes.

Within the in-house schemes, $32(47.7 \%)$ were said to operate using nurses (one of these had recently been suspended because of lack of trained staff), 12 (18\%) with optometrists, 9 (13.4\%) with orthoptists, 11 (16.4\%) had mixed personnel and in 3 schemes (4.5\%), the person contacted was unsure of which staff worked within the scheme.

Within the 18 community schemes, all but two were operated using optometrists with the remainder using general practitioners with a special interest.

\section{Secondary questionnaire survey}

Questionnaires were returned from 74 of 76 departments that stated that they operated a scheme (97\%). Nine departments admitted to not (yet) having a scheme that was actually operational, and in the remaining departments, there were 52 in-house schemes and 14 community schemes.
For the purposes of analysis, in-house schemes were divided into five subgroups, namely those who employed (1) optometrists only, (2) nurses only, (3) optometrists and either nurses or orthoptists, or both, (4) nurses and orthoptists and (5) orthoptists only. Community schemes were all considered as one group as 11 of 14 were optometrist only, with two using optometrists together with orthoptists and nurses and the final scheme operating with optometrists and orthoptists. In all the schemes, the length of staff experience tended to reflect the time the scheme had been running. Respondents often gave considerable operational details through free text.

Particulars of the operational details of the schemes broken down by principle profession of the prime workers in the scheme are shown in Table 1.

Of the optometrist-only in-house schemes (12), the longest running scheme was in Nottingham (1993), with seven commencing since the millennium. All schemes saw new and review glaucoma patients, and suspects with the Bristol scheme were the largest in all respects with 11 staff members working a total of 36 sessions per week.

Of the nurse-only in-house schemes (17), the first such scheme (Wolverhampton) commenced in 1994, with the majority starting after 2000 . In $88 \%$ of the schemes, both suspects and patients with glaucoma were seen with $12 \%$ glaucoma only. In $53 \%$ of the schemes, only review patients were seen, with $6 \%$ seeing only new patients and the remaining $41 \%$ seeing both new and review patients.

Of the in-house schemes using optometrists and others, ${ }^{5}$ the first commenced in 1996 (Hillingdon and Taunton) with $50 \%$ starting after 2000 . In $90 \%$ of the schemes, both suspects and patients with glaucoma were seen with $10 \%$ glaucoma only. In $50 \%$ of the schemes, only review patients were seen, with the remaining $50 \%$ seeing both new and review patients. In the largest scheme (Exeter), there were 16 staff members providing 20 sessions per week.

There were seven in-house schemes using nurses and orthoptists with all but one commencing after 2000. In $72 \%$ of the schemes, both suspects and those with glaucoma were seen with $14 \%$ seeing suspects only and $14 \%$ glaucoma only. In $43 \%$ of the schemes, only review patients were seen, a further $43 \%$ seeing new and review patients and $14 \%$ seeing new only.

Smallest in number were the orthoptist-only in-house schemes with the Guy's and St Thomas's being the oldest (1992) and 50\% commencing after the millennium. In 100\% of the schemes, both suspects and glaucomas were seen. In $83 \%$ of the schemes, only review patients were seen, with the remainder seeing both new and review patients.

The oldest of the 14 community schemes was from Bradford (1990) with all the others starting after the millennium. In $75 \%$ of the schemes, both suspects and 
Table 1 Shared care schemes - details of personnel and operational aspects

\begin{tabular}{|c|c|c|c|c|c|c|}
\hline & $\begin{array}{c}\text { Optom } \\
\text { only } \\
\mathrm{n}=12\end{array}$ & $\begin{array}{c}\text { Optom }+ \text { nurses/ } \\
\text { orthoptists } \\
\mathrm{n}=10\end{array}$ & $\begin{array}{l}\text { Nurse } \\
\text { only } \\
\mathrm{n}=17\end{array}$ & $\begin{array}{c}\text { Nurse }+ \\
\text { orthoptists } \\
\mathrm{n}=7\end{array}$ & $\begin{array}{c}\text { Orthoptists } \\
\text { only } \\
\mathrm{n}=6\end{array}$ & $\begin{array}{c}\text { Community } \\
\text { schemes } \\
\mathrm{n}=14\end{array}$ \\
\hline Mean training time (mo) (with range) & $19(3-48)$ & $53(0.1-120)$ & $18(6-120)$ & $24(12-36)$ & $12(3-36)$ & $6(1-24)$ \\
\hline Mean no. of staff (range) & $4(1-11)$ & $4.5(2-16)$ & $3.8(1-10)$ & $4(2-7)$ & $3.3(2-6)$ & $7.4(1-50)$ \\
\hline Mean no. of sessions per week (range) & $7.2(2-36)$ & $5.5(2-20)$ & $5.2(1-10)$ & $5.4(1-10)$ & $2.2(1-4)$ & No data \\
\hline Mean pts seen per staff member per clinic (range) & $8.8(5-12)$ & $7.5(4-10)$ & $6.0(3-10)$ & $10(3-20)$ & $6.5(4-8)$ & No data \\
\hline $\begin{array}{l}\text { Estimated \% of glaucoma care delivered by } \\
\text { scheme (range) }\end{array}$ & $5-75$ & $3-50$ & $1-80$ & $5-30$ & $1-33$ & $1-25$ \\
\hline $\begin{array}{l}\text { Mean estimated \% of visits requiring doctor's } \\
\text { input (range) }\end{array}$ & $26(10-80)$ & $10(7-13)$ & $12(1-20)$ & $18(10-25)$ & $9(1-15)$ & $23(4-90)$ \\
\hline$\%$ of schemes with doctor available to give opinion & 83 & 100 & 94 & 100 & 83 & 21 \\
\hline$\%$ of schemes with regular review by doctor & 42 & 50 & 53 & 57 & 67 & 58 \\
\hline $\begin{array}{l}\% \text { of schemes in which doctor regularly reviews } \\
\text { records }\end{array}$ & 17 & 50 & 17 & 83 & 83 & 67 \\
\hline$\%$ of schemes with regular audit & 67 & 70 & 71 & 86 & 100 & 92 \\
\hline
\end{tabular}

Abbreviations: mo, months; no, number; optom, optometrists; pts, patients.

First five columns are hospital-based schemes.

Table 2 Activities performed by shared care staff in schemes, figures are percentages

\begin{tabular}{lrrrrrrrrrr}
\hline Type of scheme & Acuity & IOP & Gonio & Fields & Ant Seg & Fundus & Imaging & Interpret & Decisions & Prescribing \\
\hline In-house Optoms $n=12$ & 58 & 100 & 71 & 33 & 100 & 100 & 42 & 100 & 83 & 8 \\
In-house Optoms + others $n=10$ & 100 & 100 & 40 & 100 & 100 & 100 & 90 & 90 & 60 & 10 \\
In-house Nurses $n=17$ & 100 & 100 & 50 & 29 & 94 & 71 & 65 & 88 & 59 & 29 \\
In-house Nurses + orthop $n=7$ & 86 & 100 & 14 & 86 & 86 & 86 & 57 & 86 & 57 & 0 \\
In-house orthoptists $n=6$ & 100 & 100 & 33 & 100 & 83 & 33 & 83 & 86 & 50 & 17 \\
Community schemes $n=12$ & 100 & 100 & 25 & 100 & 100 & 92 & 58 & 92 & 33 & 0 \\
\hline
\end{tabular}

Abbreviations: Optoms, optometrists; Orthop, orthoptists; Gonio, gonioscopy performed, Ant Seg, anterior segment examination performed; Interpret, interpretation of results.

glaucomas were seen, with $17 \%$ seeing glaucomas only and $8 \%$ suspects only. In $58 \%$ of the schemes, only review patients were seen, with $25 \%$ seeing new and review patients and $8.5 \%$ new only (one non-responder). No meaningful data were available for the number of sessions per week or the mean number of patients seen per clinic per staff member. This is probably because of the 'modus operandi' of most community optometric schemes, in which patients were interspersed with their usual clients.

Table 2 shows the tests and examinations carried out by the shared care staff in the different types of schemes along with the level of management responsibility expected of the staff.

\section{Discussion}

Peer-reviewed literature examining the efficacy or safety of the utilisation of non-ophthalmologists in the management of glaucoma and glaucoma suspects in the United Kingdom is limited to a few schemes, mostly involving optometrists. ${ }^{6-9}$ Our study, which was carried out before the results of the Department of Health pilot schemes from community care in glaucoma were published, shows that many departments had developed one or more schemes well in advance of this. A strength of our study is that responses were obtained from $100 \%$ of the units; however, a possible bias reducing the incidence of schemes is the possibility that the telephone contact was unaware of an existing scheme within the department. As with any questionnaire, misinterpretation of questions may have also biased the results, and not all respondents answered all questions and the results were given as percentages of those who responded.

This study, the first to identify the number and nature of shared care schemes involving the management of glaucoma and glaucoma suspects in the United Kingdom, does however, offer a 'snapshot-in-time' review of the operational characteristics of English schemes that operated in May 2006. Almost all the schemes saw patients with glaucoma and were therefore not restricted to glaucoma suspects. Care must be taken not to extrapolate data from this study to those countries not studied or to assume that the scheme numbers and operational details have remained unchanged. 
It is clear from Table 2 that the staff members were not interpreting the results of the tests in all the schemes, a 'sine-qua-non' of our definition of shared care. ${ }^{1}$ However, more than $85 \%$ of the schemes in each category involve paramedical staff in the interpretation of data, with a significant proportion making management decisions and a small number prescribing without recourse to medical opinion (Table 1). Variations in the operational activity of the carers seem to occur with scheme subtype. In the community schemes, in which optometrists predominate and the presence of a doctor is rare, only one-third make management decisions, whereas optometrists working in an in-house scheme make management decisions in $83 \%$ of the schemes. It seems likely that the presence of a doctor available for consultation, if necessary, encourages the development of decision-making skills within shared care schemes with the opposite also being true.

We do not consider it of value to perform statistical analysis of the results of the survey in view of the estimates required by the respondents to many of the questions. However, it would seem that optometrists tend to see more patients per session and are more likely to examine the fundus (and therefore the optic disc) in the in-house clinics than the other health-care professionals performing shared care duties, and they are also more likely to see new patients. This probably reflects the optometrists' greater experience in examination techniques relevant for glaucoma before undergoing specialist training for glaucoma shared care.

A smaller number of patients seen per session does not necessarily indicate reduced efficiency, as, although the 'orthoptists-only' group sees only an average of 6.5 patients per session, they perform visual fields in all the schemes (the 'in-house-optometrists' only and 'nursesonly' schemes presumably relying on others to provide them with the fields to interpret).

The large range of training times perhaps illustrates the diversity of the experience of the staff members working in the schemes, but also probably relates to the variable complexity of the schemes and to the different roles scheme members are expected to play in the management process. For example in one scheme, operated by optometrists and managing ocular hypertensives in the community, the training period was only 3 days. In this scheme, each patient has an IOP 'ceiling' allowable before return to the hospital is mandatory, the level being pre-determined by the glaucoma consultant; the only other two decisions required by the shared care optometrists being is the field normal' and 'has the optic disc changed from the baseline stereo photograph'. In contrast, in an 'in-house' scheme, also using optometrists, the complexity of the patients seen by the shared care staff is controlled, increasing with experience and competency on an individual basis with an 'apprenticeship model' training taking up to 4 years.

The questionnaire responses are of interest for reasons of clinical governance. It is interesting to note that in 14 of the $30(47 \%)$ schemes seeing new patients, gonioscopy is not performed and in 9 of these, a management decision is expected from the shared care staff, with one prescribing without gonioscopy or a doctor's review. Such a practice does not follow the RCOphth guidelines for glaucoma and ocular hypertension operating at the time of the study ${ }^{10}$ or those from the European Glaucoma Society, which describes gonioscopy as 'a fundamental part of the comprehensive eye examination' ${ }^{5}$ Other clinical governance-related issues include the absence of a regular audit in many schemes, particularly those running 'in-house' (Table 1).

Thus, it is clear that there is a wide diversity of operational details within the shared care schemes in glaucoma operating in England. The majority of schemes are relatively new and see only a small number of patients per year. A few are better established and provide a major part of the glaucoma care for the area served. Fine details of even the larger schemes were considered outside the scope of this short report, but operational and audit data from these larger schemes would be of value to those departments considering investing in a shared care scheme and we encourage the supervisors to publish such data. One such communitybased scheme has recently done this, ${ }^{11}$ creating a plethora of concerns from ophthalmologists interested in glaucoma care (R Bourne, personal communication, November 2008). Chronic glaucoma care is currently under review by NICE (National Institute of Clinical Excellence). Schemes may well have to adapt to be acceptable under the new NICE guidelines. A repeat study after adoption of the guidelines is planned.

\section{Acknowledgements}

This work has been funded by an unconditional grant from the RNIB.

\section{References}

1 Department of Health. Evaluation of the chronic eye care services programme: final report. January 2007, http:// www.aop.org.uk/uploaded_files/dh_evaluation_report of_pilots_jan_2007.pdf.

2 Department of Health. National Eye Care Services Group - first report. Department of Health: London, April 2004, http://www.aop.org.uk/uploaded_files / first_report_of_group_from_dh.pdf.

3 Hitchings RA. Shared care for glaucoma. Br J Ophthalmol 1995; 79: 626 
4 Royal College of Ophthalmologists. Directory of Training Posts 2005/6.

5 Terminology and Guidelines for Glaucoma. 2nd edn. European Glaucoma Society 2003. ISBN: 88-87434-13-1.

6 Henson DB, Spencer AF, Harper R, Cadman EJ. Community refinement of glaucoma referrals. Eye 2003; 17: 21-26.

7 Banes M, Culham MC, Bunce C, Xing W, Viswanathan A, Garway-Heath D. Agreement between optometrists and ophthalmologists on clinical management decisions for patients with glaucoma. $\mathrm{Br} J$ Ophthalmol 2006; 90: $579-585$.
8 Johnson ZK, Griffiths PG, Birch MK. Nurse prescribing in glaucoma. Eye 2003; 17: 47-52.

9 Spry PGD, Spencer ID, Sparrow JM, Peters PJ, Brookes ST, Gray $\mathrm{S}$ et al. The Bristol shared care glaucoma study: reliability of community optometric and hospital eye services test measures. Br J Ophthalmol 1999; 83: 707-712.

10 Management of Open Angle Glaucoma and Ocular Hypertension. Royal College of Ophthalmologists 2003.

11 Gupta D, Raines M, Roy K. Results of a pilot optician-led stable glaucoma follow-up scheme. Optometry Today 2008; $40-44$.

Supplementary Information accompanies the paper on Eye website (http://www.nature.com/eye) 